\title{
Sintomas gastrointestinais em pacientes com COVID-19: relação com a gravidade e complicações da doença
}

\author{
Gastrointestinal symptoms in patients with COVID-19: relationship with severity and \\ complications of the disease
}
Síntomas gastrointestinales en pacientes con COVID-19: relación con la gravedad y las complicaciones de la enfermedad

\begin{abstract}
Mariana Yumi Ogassawara ${ }^{1 *}$, Nestor Augusto Avelino Leite ${ }^{2}$, Debora Zandrovski Gonçalves ${ }^{3}$, Ananda Saunders Fernandes Santos ${ }^{4}$, Victor Antonio Dal Col Silva ${ }^{5}$, Tiago Picolo Fernandes ${ }^{6}$, Gabrielly Kaiumy Otani Prestes Cardoso Wagner ${ }^{6}$, Jennifer Nicole Padua Kinsler ${ }^{5}$, Isabella Angeli Pahim $^{7}$, Estéfany Kotaka Munhoz ${ }^{8}$.
\end{abstract}

\begin{abstract}
RESUMO
Objetivo: Revisar as manifestações do comprometimento gastrointestinal, hepático e pancreático da COVID19, abordando, junto aos mecanismos fisiopatológicos, a incidência, sintomas, complicações, diagnóstico e manejo destes pacientes. Revisão bibliográfica: A doença do novo coronavírus se disseminou rapidamente pelo mundo, resultando em uma pandemia global. Embora seja uma doença de caráter predominantemente respiratório, entre $39 \%$ e $61 \%$ dos pacientes infectados apresentam sintomas gastrointestinais, sendo os principais diarreia, náuseas, vômitos e dor abdominal. Em alguns casos, pacientes podem manifestar sintomas gastrointestinais exclusivamente, sem queixas respiratórias. Comprometimento hepático é comum, sendo observado aumento de enzimas hepáticas em $30 \%$ das pessoas com infecção confirmada por COVID19 , o que pode ser utilizado como marcador de gravidade e preditor de mortalidade. Estudos relacionam a presença de sintomas gastrointestinais com formas mais graves da COVID-19 e um pior prognóstico. Considerações finais: Conclui-se que há relação significativa de infecção pelo Sars-Cov-2 e sintomas gastrointestinais, principalmente em pacientes graves. Diante disso, é importante que queixas gastrointestinais não sejam ignoradas. Contudo, mais estudos precisam ser feitos para melhor determinação da prevalência de sintomas gastrointestinais na COVID-19, visto ser uma patologia recente.
\end{abstract}

Palavras-chave: Infecção pelo Coronavírus 2019-nCoV, Trato gastrointestinal, Sinais e sintomas.

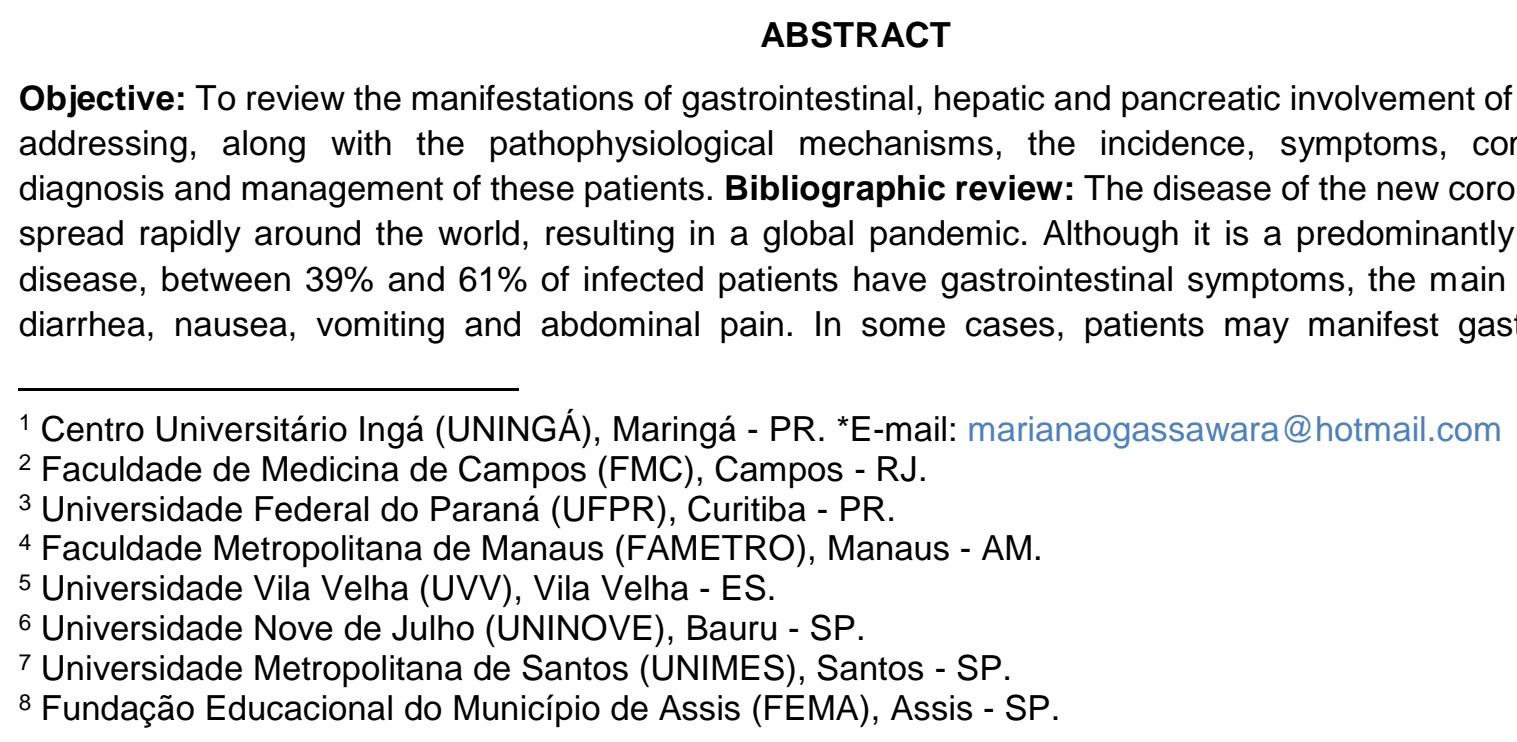


symptoms exclusively, without respiratory complaints. Hepatic impairment is common, with an increase in liver enzymes observed in $30 \%$ of people with confirmed infection by COVID-19, which can be used as a marker of severity and a predictor of mortality. Studies have linked the presence of gastrointestinal symptoms with more severe forms of COVID-19 and a worse prognosis. Final considerations: We conclude that there is a significant relationship between infection by Sars-Cov-2 and gastrointestinal symptoms, especially in critically ill patients. Therefore, it is important that gastrointestinal complaints are not ignored. However, more studies need to be done to better determine the prevalence of gastrointestinal symptoms in COVID-19, as it is a recent pathology.

Keywords: 2019-novel Coronavirus (2019-nCoV) infection, Gastrointestinal tract, Signs and symptoms.

\section{RESUMEN}

Objetivo: Revisar las manifestaciones de afectación gastrointestinal, hepática y pancreática del COVID-19, abordando, junto con los mecanismos fisiopatológicos, la incidencia, síntomas, complicaciones, diagnóstico y manejo de estos pacientes. Revisión bibliográfica: La enfermedad del nuevo coronavirus se ha extendido rápidamente por todo el mundo, llevando a una pandemia mundial. Aunque se trata de una enfermedad predominantemente respiratoria, entre $39 \%$ y $61 \%$ de los pacientes infectados presentan síntomas gastrointestinales, siendo los principales diarrea, náuseas, vómitos y dolor abdominal. En algunos casos, los pacientes pueden manifestar síntomas gastrointestinales exclusivamente, sin molestias respiratorias. La insuficiencia hepática es común, observándose un aumento de las enzimas hepáticas en el $30 \%$ de las personas con infección confirmada por COVID-19, que puede utilizarse como marcador de gravedad y predictor de mortalidad. Los estudios han relacionado la presencia de síntomas gastrointestinales con formas más graves de COVID-19 y un peor pronóstico. Consideraciones finales: Concluimos que existe una relación significativa entre la infección por Sars-Cov-2 y los síntomas gastrointestinales, especialmente en pacientes críticamente enfermos. Por lo tanto, es importante que no se ignoren las molestias gastrointestinales. Sin embargo, es necesario realizar más estudios para determinar mejor la prevalencia de síntomas gastrointestinales en COVID-19, ya que es una patología reciente.

Palabras clave: Infección por Coronavirus 2019-nCoV, Tracto gastrointestinal, Signos y síntomas.

\section{INTRODUÇÃO}

A doença do novo coronavírus, conhecida popularmente como COVID-19, foi descoberta em dezembro de 2019, em Wuhan, na China. Causada pela síndrome respiratória aguda grave coronavírus 2 (Sars-CoV2), a COVID-19 se disseminou rapidamente por todo o mundo, resultando em uma pandemia global, declarada pela OMS em 11 de março de 2020 (OBA J, et al., 2020).

As manifestações clínicas do COVID-19, embora sejam predominantemente relacionadas ao sistema respiratório, são bem marcantes em outros sistemas orgânicos, como o trato gastrointestinal. Estima-se que cerca de um terço dos pacientes infectados pelo Sars-CoV-2 apresentem sintomas gastrointestinais, como náuseas, vômitos, diarréia, anorexia, inapetência e dor abdominal. Mialgia e fadiga também são comumente relatados, enquanto os distúrbios gustativos e olfativos são mais comuns quando associados a outras manifestações (KAAFARANI HMA, 2020).

Um estudo publicado em janeiro de 2020 relatou um paciente com diarreia como uma manifestação gastrointestinal (GI) da infecção por SARS-CoV-2. Desde então, vários casos relatando a doença do coronavírus associada a sintomas gastrointestinais, como já relatados, foram descritos. Além disso, os estudos mostraram casos graves com a presença de SARS-CoV-2 em úlceras esofágicas, bem como no estômago, duodeno e tecidos retais. Também foi observado que pacientes que apresentam a manifestação grave da doença possuem uma maior probabilidade de ter dor abdominal, quando comparados a pacientes não graves, bem como maior chance de ter níveis séricos elevados de biomarcadores relacionados à função e lesão hepática, os quais estão associados ao envolvimento de sintomas Gl e pior prognóstico da doença (SILVA FAF, et al., 2020). 
Outro estudo, sendo esse um estudo de coorte, foi realizado com 2.477 pacientes positivos para SarsCoV-2, entre esses pacientes 7,8\% deles apresentaram diarréias, $5,5 \%$ náuseas e/ou vômitos e apenas de 2 a $5 \%$ dor abdominal (SURESH KUMAR VC, et al., 2020).

A fisiopatologia que explica esses sintomas ainda é pouco esclarecida, mas sabe-se da sua relação com o receptor da enzima conversora de angiotensina 2 (ECA2), o qual se encontra em grande quantidade no sistema gastrointestinal. Acredita-se que esse receptor funcione como porta de entrada para o vírus, que se liga a ele e penetra a célula, podendo lesionar diretamente os órgãos desse sistema. Além disso, estudos indicam que o vírus causador da doença COVID-19 pode permanecer nas fezes por muito tempo, até mesmo depois de cessar a colonização do vírus no sistema respiratório, o que caracteriza uma transmissão oral-fecal (AMARAL LT, et al., 2020; SURESH KUMAR VC, et al., 2020).

Sendo assim, torna-se necessário uma maior atenção para as manifestações gastrointestinais decorrentes da doença COVID-19 para que não passem despercebidas, visto que são diferentes dos sintomas respiratórios, que são os mais frequentes. Por conseguinte, diante desse contexto, o presente artigo tem como objetivo abordar e revisar conceitos, junto aos mecanismos fisiopatológicos, referente às manifestações de sintomas gastrointestinais em pacientes infectados pelo Sars-CoV-2.

\section{REVISÃO BIBLIOGRÁFICA}

\section{Conceito e relação dos sintomas com a gravidade do COVID-19}

Segundo a Organização Mundial da Saúde (OMS) o SARS-CoV-2 é um agente infeccioso associado a uma apresentação clínica de amplo espectro, que classicamente envolve sintomas do trato respiratório, como febre, tosse seca e falta de ar. A realização de novos estudos possibilitou a relação de sintomas gastrointestinais aos quadros clínicos de COVID-19 (HOU XH, 2020; FANG D, et al., 2020).

Pacientes gravemente afetados têm maior probabilidade de apresentar quadros de dor abdominal e comprometimento da função hepática, consequentemente obtendo um pior prognóstico da doença (SURESH KUMAR VC, et al., 2020; MAO R, et al., 2020). O aumento das transaminases é considerado um marcador de gravidade da doença e preditor de mortalidade gerando grande preocupação, uma vez que quase dois terços dos pacientes com complicações graves de COVID-19 apresentam transaminases hepáticas elevadas (ZHAO X, et al., 2021; ZHANG C, et al., 2020).

O prognóstico dos pacientes com COVID-19 e sintomas gastrointestinais ainda é desconhecido (MAO R, et al., 2020) contudo, estudos também sugerem que pacientes com sintomas gastrointestinais apresentam maiores complicações em relação aos pacientes sem sintomas gastrointestinais (JIN X, et al., 2020).

\section{Sintomas}

Cerca de um terço dos pacientes contaminados com o vírus da COVID-19 apresentam queixas gastrointestinais (HAYTHAM M, et al., 2021). Em pacientes adultos, as mais comuns são diarreia, seguida por náuseas / vômitos e dor abdominal, enquanto em pacientes pediátricos, vômitos são relatados com mais frequência. Além desses sintomas, a perda de apetite, anosmia, ageusia e sangramento intestinal também foram relatados, porém em número menor de casos (SILVA FAF, et al., 2020).

Alguns pacientes podem manifestar sintomas gastrointestinais na ausência de queixas respiratórias, sendo principalmente a diarréia o primeiro sintoma, com ausência de desidratação ou hematoquezia. A ocorrência da mesma é descrita como fezes amarela e pastosa fina ou aquosa, com frequência de três a nove dejeções ao dia (OBA J, et al., 2020; ZHANG H, et al., 2020).

Foi analisado também que pacientes com sintomas gastrointestinais são mais propensos a desenvolverem a forma grave da COVID-19 em comparação aos pacientes assintomáticos, causando sintomas específicos e alterações laboratoriais, como níveis séricos anormais de enzimas hepáticas, contribuindo para pior prognóstico da doença (ZHANG H, et al., 2020; SILVA FAF, et al. 2020).

Conforme Suresh Kumar VC, et al. (2020), que analisou 17 outros estudos, a dor abdominal se apresenta como o sintoma gastrointestinal de maior indicativo para gravidade de COVID-19, estando presente em 8,59\% 
dos casos graves e em apenas $0,8 \%$ dos casos leves, tendo uma diferença de quase 8 pontos percentuais de distinção que podem colaborar para que o profissional se atente previamente para o possível agravamento do quadro. Sendo seguida pela ocorrência de diarréia, manifestada por $11,07 \%$ dos pacientes graves e 6,24\% dos pacientes leves, obtendo quase 5 pontos percentuais, o que também pode colaborar, embora em menor grau, com a escolha da conduta a ser seguida. Já a ocorrência de náusea ou vômito não se apresentou como um grande fator diferencial, pois menos de 2 pontos percentuais distanciam a ocorrência de tais sintomas entre os dois grupos (7,59 em graves e 6,29 em leves) (SURESH KUMAR VC, et al., 2020).

É possível que a lesão dos enterócitos intestinais causam disfunção do íleo e do cólon, levando à má absorção, desequilíbrio na secreção intestinal e ativação do sistema nervoso entérico, resultando na presença da diarreia (DÍAZ LA e ESPINO A, 2020; CHA MH, et al., 2020; HAYASHI Y, et al., 2021).

\section{Complicações}

Várias complicações gastrintestinais foram relatadas em pacientes gravemente enfermos com COVID-19. É provável que essa alta incidência de complicações não seja apenas uma manifestação geral de doença crítica, mas parcialmente atribuível à própria infecção viral (KAAFARANI HMA, et al., 2020). Fatores de risco potenciais nestes pacientes incluem doenças inflamatórias crônicas, como comorbidades e o uso de glicocorticóides (SUNANDA VK, et al., 2021).

Durante a hospitalização prolongada dos pacientes em estado crítico, as complicações variam de transaminite autorresolvente e intolerância alimentar a isquemia mesentérica com risco de vida (KAAFARANI HMA, et al., 2020). Dentre elas, a mais grave é a isquemia mesentérica. Os pacientes que são admitidos na UTI podem desenvolver a doença simplesmente devido a altas doses de vasopressores, instabilidade hemodinâmica e distúrbios metabólicos que comprometem o fluxo sanguíneo intestinal, contribuindo para uma alta taxa de mortalidade (KAAFARANI M, et al., 2020).

O envolvimento hepático de SARS-CoV-2 pode ser explicado por um dano transitório ao hepatócito. Estudos descobriram que a maioria das lesões hepáticas são leves e transitórias, mas também podem ocorrer lesões hepáticas graves. De acordo com o American College of Gastroenterology (ACG), enzimas hepáticas alteradas, como aumento da aspartato e alanina transferase e da bilirubina total, são observadas em $30 \%$ das pessoas com infecção confirmada por COVID-19 (PATEL KP, et al., 2020; DÍAZ LA e ESPINO A, 2020; $\mathrm{CHA} \mathrm{MH}$, et al., 2020).

As alterações no perfil hepático de pacientes com diagnóstico confirmado de COVID-19 foram classificadas como: aumento das transaminases maior que três vezes o limite superior, sendo um marcador de gravidade é um preditor independente de mortalidade; aumento da fosfatase alcalina e gama-glutamil transferase duas vezes acima do limite superior; uso de medicamentos de terapia antiviral que podem causar danos ao fígado, como o lopinavir/ritonavir; e dano hepático causado diretamente pelo vírus (DIAZ CFF e TABOADA OYZ, 2020; DÍAZ LA e ESPINO A, 2020; KAAFARANI HMA, et al., 2020).

Estudos recentes documentaram níveis elevados de amilase e lipase sérica em pacientes infectados, sugerindo a possibilidade de dano pancreático decorrente da doença (DIAZ CFF e TABOADA OYZ, 2020). Os mecanismos pelos quais a lesão pancreática pode ocorrer incluem os efeitos citopáticos diretos do SARSCoV-2, ou respostas celulares inflamatórias sistêmicas indiretas e imunomediadas, resultando em dano a órgãos ou anormalidades enzimáticas secundárias (KISHAN P, et al., 2020). A colecistite aguda e a pancreatite aguda foram relatadas especialmente nos pacientes gravemente enfermos (KAAFARANI HMA, et al., 2020).

Outra complicação relatada por alguns estudos é a pseudo-obstrução colônica aguda, uma síndrome colônica distinta no paciente criticamente doente, caracterizada por distensão gasosa grave sem obstrução distal óbvia (KAAFARANI HMA, et al., 2020). Além disso, existem outras condições inflamatórias crônicas, como a doença inflamatória intestinal (DII), que apresentam um risco de tornar o indivíduo mais suscetível ao COVID-19 ou a um curso mais grave da doença. A doença ativa leva a um resultado negativo que requer hospitalização mais longa e ventilação assistida (KARIYAWASAM CJ, et al., 2021). 


\section{Faixa etária e incidência dos sintomas}

Sendo importante ressaltar que à medida que novos estudos são realizados em pacientes com COVID19, os sintomas gastrointestinais ficam mais evidentes, os mais comuns são: diarreia, náusea, dor abdominal e vômitos. Um estudo realizado em 2019, analisou dados epidemiológicos, clínicos, demográficos e laboratoriais de 95 paciente que foram infectados pelo SARS-CoV-2. com média de idade 45,3 $\pm 18,3$ anos, dentre eles, 58 casos apresentaram sintomas gastrointestinais na admissão ou no curso da doença. Dessa forma, segundo este estudo, a prevalência foi de que $61 \%$ dos acometidos pela doença apresentaram manifestações gastrointestinais (LIN L, et al., 2020).

Além disso, foi realizado em 2020 um estudo com 140 pacientes da China com diagnóstico confirmado de infecção viral por SARS-CoV-2. Neste estudo, a média de idade geral dos infectados foi de 57,0 anos e a proporção entre homens e mulheres foi praticamente equivalente. Assim, dentre estes pacientes, 39,6\% apresentaram manifestações gastrointestinais (ZHANG JJ, et al., 2020).

Portanto, mesmo que ainda existam variações nos resultados destes estudos, pode-se concluir que ao contrário do que se pensava no início da pandemia, existe uma relevante incidência dos sintomas gastrointestinais em pacientes com COVID-19. Desta forma, cabe uma maior atenção dos médicos para estas manifestações gastrointestinais em pacientes infectados pelo SARS-CoV-2 (AMARAL LTW, et al., 2020).

\section{Diagnóstico}

Sabe-se que alguns pacientes infectados pela COVID-19 tiverem sintomas gastrointestinais variados, incluindo, diarréia, constipação, náusea, vômitos, dor epigástrica e melena. Dos indivíduos que manifestaram sintomas, muitos tiveram complicações baixas e moderadas, principalmente com diarreia durante o período da doença. Deste modo, a diarreia é parâmetro de consciência e necessita de análise investigativa para diagnóstico precoce. Além disso, a calprotectina com suas dosagens oferece importante monitoramento, diagnóstico e assistência da diarreia associada ao Sars-CoV-2 e agravamentos gastrointestinais (SAHU T, et al., 2021).

Em dias atuais, identificando sintomas gastrointestinais como apresentação do quadro inicial do paciente, o teste fecal é benéfico para teste da COVID-19, o diagnóstico rápido é importante para a detecção precoce do vírus. O uso de testes de ácido nucléico fecal tem aumentado, esse teste é o método recomendado para o diagnóstico nas fezes, realizando por método de técnica quantitativa de reação em cadeia polimerase em tempo real (PCR) (qPCR). Estudos de Zhang JC, et al. (2020) revelaram que amostras fecais eram precisas igualmente as amostras da faringe que já são frequentemente utilizadas e que o teste de fezes positivo não tem relação com a gravidade da contaminação pulmonar. As vantagens do teste fecal é a redução de contaminação da equipe médica quando comparada com o método de esfregaço orofaríngeo, entretanto, endoscopia e colonoscopia no diagnóstico do Sars-CoV-2 permanecem desconhecidos (PATEL KP, et al., 2020).

Dados laboratoriais são dignos de nota em pacientes infectados pelo novo coronavírus, em $20 \%$ a $30 \%$ dos indivíduos possuem elevação dos níveis de aminotransferase, também sendo observado hipoproteinemia e elevação do tempo de protrombina. Queda de monócitos e prevalência de antibioticoterapia presentes em pacientes adultos, em crianças, linfopenia, mau indicador de prognóstico, é incomum. Proteína C-reativa, citocinas, ferritina, procalcitonina, dímeros $D$ e triglicérides foram incluídas em casos de choque hiper inflamatório. As identificações laboratoriais podem avaliar a gravidade da infecção. (OBA J, et al., 2020).

\section{Manejo do paciente}

É importante que o profissional permaneça atento aos sintomas gastrointestinais que o paciente venha a apresentar, não deixando-os de lado, subestimando ou desconsiderando-os, visto que existe a possibilidade de sintomatologia exclusivamente gastrointestinal da COVID-19, mesmo que em baixa incidência (GALANOPOULOS M., et al., 2020). Tais cuidados devem ser mantidos a fim de evitar e reduzir a exposição e o risco de infecção durante a realização de procedimentos, tais como a endoscopia, sendo imprescindível o uso correto dos equipamentos de proteção individual durante o manejo do paciente (KANE, S. V., et al., 2021; KARIYAWASAM JC, et al., 2021). Ademais, o predomínio de sintomas de "gastroenterite" deve levar à suspeita imediata de infecção por SARS-CoV-2 (GALANOPOULOS M, et al., 2020). 
Também deve-se considerar a baixa especificidade dos sintomas gastrointestinais prevalentes, já que também podem se manifestar sem que haja infecção por SARS-CoV-2, o que reforça, novamente, o quão relevante é a manter uma suspeita prévia de COVID-19 para a obtenção do diagnóstico precoce e redução de casos ativos, não se excluindo a hipótese de infecção nem mesmo para pacientes em acompanhamento de doenças gastrointestinais pré-existentes (GALANOPOULOS M, et al., 2020).

E devido à maior incidência de casos graves em pacientes com COVID-19 que apresentam sintomas gastrointestinais, é essencial que tal suposição seja verificada o quanto antes, para que se possa atuar no caso antes da evolução do quadro (ALMEIDA JFM e CHEHTER EZ, 2020). Um dos fatores que colaboram para essa prioridade é a ausência de tratamento específico para a COVID-19, pois quanto antes é feita a identificação, mais efetivo é o isolamento, o que diminui a transmissibilidade tanto em nível familiar como hospitalar (AMARAL LTW, et al., 2020).

O tratamento de escolha deve ser baseado na supressão dos sintomas e nos cuidados de suporte, já que as manifestações gastrointestinais tendem a desaparecer com o tempo, não se agravando na maior parte dos casos. Deve-se, portanto, favorecer o bem-estar do paciente, fornecendo analgésicos e antieméticos, e manter adequadas a oxigenação e a hidratação, bem como o equilíbrio eletrolítico, a fim de evitar possíveis distúrbios. No caso de manifestações mais graves deve-se avaliar cada caso com o auxílio de uma equipe multidisciplinar, o que permite uma melhor e mais específica abordagem (KARIYAWASAM JC, et al., 2021).

\section{CONSIDERAÇÕES FINAIS}

Através da análise bibliográfica não sistemática desse estudo, conclui-se que há relação significativa de sintomas gastrintestinais e infecção pelo Sars-Cov-2. A quantidade e gravidade dos sintomas gastrintestinais estão diretamente relacionados ao pior prognóstico da COVID-19. As manifestações gastrintestinais incluem diarreia, vômito, náusea e dor abdominal, além de perda de apetite e sangramento gastrointestinal. Podem ser relatados também comprometimento hepático e pancreático, principalmente nos pacientes que necessitam de hospitalização. Diante disso, é importante que queixas gastrintestinais não sejam ignoradas, principalmente por existir a possibilidade de sintomatologia gastrintestinal exclusiva. Contudo, mais estudos ainda precisam ser feitos para melhor identificação da prevalência de sintomas gastrintestinais na COVID-19, visto ser uma patologia recente.

\section{REFERÊNCIAS}

1. ALMEIDA JFM, CHEHTER EZ. COVID-19 e o trato gastrointestinal: o que já sabemos?. Einstein (São Paulo), 2020; 18: eRW5909.

2. AMARAL LTW, et al. Sintomas abdominais como manifestação inicial da COVID-19: uma série de casos. Einstein (São Paulo), 2020; 18: eRC5831.

3. CHA MH, et al. Gastrointestinal and hepatic manifestations of COVID-19: A comprehensive review. World Journal of Gastroenterology, 2020; 26(19): 2323-2332.

4. DIAZ CFF, Taboada OYZ. Manifestaciones gastrointestinales de la infección por el "nuevo coronavirus". Revista Colombiana de Gastroenterología, 2020; 35(Supl 1): 69-72.

5. DíAZ LA, Espino A. Manifestaciones gastrointestinales de pacientes infectados con el nuevo Coronavirus SARS-CoV2. Revista Gastroenterología Latinoamericana, 2020; 31(1): 35-38.

6. FANG D, et al. Manifestations of digestive system in hospitalized patients with novel coronavirus pneumonia in Wuhan, China: a single-center, descriptive study. Chinese Journal of Digestion, 2020; (12): e005-e005.

7. GALANOPOULOS M, et al. COVID-19 pandemic: Pathophysiology and manifestations from the gastrointestinal tract. World Journal of Gastroenterology, 2020; 26(31): 4579-4588.

8. HAYASHI Y, et al. The characteristics of gastrointestinal symptoms in patients with severe COVID-19: a systematic review and meta-analysis. Journal of Gastroenterology, 2021; 56: 409-420.

9. HAYTHAM M, et al., 2021. COVID-19: Gastrointestinal symptoms and complications. UpToDate, 2021; 129171(1): 114.

10. HOU XH. Attention to the damage of the new 2019 coronavirus to the digestive system and the possibility of fecal-oral transmission. Chinese Journal of Digestion, 2020; 40(03): 145-147.

11. ISER BPM, et al. Definição de caso suspeito da COVID-19: uma revisão narrativa dos sinais e sintomas mais frequentes entre os casos confirmados. Epidemiologia e Serviços de Saúde, 2020; 29(3): e2020233

12. JIN X, et al. Epidemiological, clinical and virological characteristics of 74 cases of 2019 coronavirus-infected disease (COVID-19) with gastrointestinal symptoms. Gut, 2020; 69(6): 1002-1009. 
13. KAAFARINI HMA, et al. Gastrointestinal Complications in Critically III Patients With COVID-19. Annals of Surgery, 2020; 272(2): e61-e62.

14. KANE SV, et al. COVID-19: Issues related to gastrointestinal disease in adults. UpToDate, 2021; $127965(19): 1-17$.

15. KARIYAWASAM JC, et al. Gastrointestinal manifestations in COVID-19. Transactions of The Royal Society of Tropical Medicine and Hygiene, 2021; 0: trab042.

16. LIN L, et al. Gastrointestinal symptoms of 95 cases with SARS-CoV-2 infection. Gut, 2020; 69(6): 997-1001

17. MAO R, et al. Manifestations and prognosis of gastrointestinal and liver involvement in patients with COVID-19: a systematic review and meta-analysis. Lancet Gastroenterology \& Hepatology, 2020; 5(7): 667-678.

18. OBA J, et al. Sintomas gastrintestinais e abordagem nutricional durante a pandemia de COVID-19: guia prático para pediatras. Einstein (São Paulo), 2020; 18: eRW5774.

19. PATEL KP, et al. Gastrointestinal, hepatobiliary, and pancreatic manifestations of COVID-19. Journal of Clinical Virology, 2020; 128: 104386.

20. SAHU T, et al. Current understanding of the impact of COVID-19 on gastrointestinal disease: Challenges and openings. World Journal of Gastroenterology, 2021; 27(6): 449-469.

21. SILVA FAF, et al. COVID-19 gastrointestinal manifestations: a systematic review. Revista da Sociedade Brasileira de Medicina Tropical, 2020; 53: e20200714.

22. SUNANDA VK, et al. COVID-19: Issues related to gastrointestinal disease in adults. UpToDate, 2021; 127965(19): 117.

23. SURESH VC, et al. Novelty in the gut: a systematic review and meta-analysis of the gastrointestinal manifestations of COVID-19. BMJ Open Gastroenterology, 2020; 7(1): e000417.

24. ZHANG C, et al. Liver injury in COVID-19: management and challenges. Lancet Gastroenterology \& Hepatology, 2020; 5(5): 428-430.

25. ZHANG H, et al. Specific ACE2 expression in small intestinal enterocytes may cause gastrointestinal symptoms and injury after 2019-nCoV infection. International Journal of Infection Diseases, 2020; 96:19-24.

26. ZHANG JJ, et al. Clinical characteristics of 140 patients infected with SARS-CoV-2 in Wuhan, China. European Journal of Allergy and Clinical Immunology, 2020; 75(7): 1730-1741.

27. ZHANG JC, et al. Fecal specimen diagnosis 2019 novel coronavirus-infected pneumonia. Journal of Medical Virology, 2020; 92(6): 680-682.

28. ZHAO X, et al. The impact of coronavirus disease 2019 (COVID-19) on liver injury in China: A systematic review and meta-analysis. Medicine (Baltimore), 2021; 100(4): e24369. 